\title{
SNAPVis and SPANVis: Ontologies for Recognizing Variable Vista Spatial Environments
}

\author{
Tiansi Dong \\ Cognitive Systems Group, \\ Department of Math and Informatics, University of Bremen, \\ 28334 Bremen, Germany \\ tiansi@informatik.uni-bremen.de
}

\begin{abstract}
This paper gives the SNAP and SPAN ontologies relating to recognizing variable vista spatial environments, namely, SNAPVis and SPANVis. It proposes that recognizing spatial environments is a judgment process of whether the perceived environment is compatible with the remembered one. Their compatibility is based on both their spatial changes and the commonsense knowledge of objects' stabilities. The recognition result is determined by whether such changes are due to possible movements of related objects or not. This paper presents six SNAPVis ontologies: fiat boundary, near region, fiat parts (the three are fiat regions), classic topologic relations, qualitative orientations, and qualitative distances (the three are spatial relations) and one SPANVis ontology: the commonsense knowledge of stability of spatial objects. The paper briefly presents a cognitive map of vista spatial environments and the process of recognition.
\end{abstract}

\section{An Introduction}

"The structure of space can be described from the point of view of behavior", cf. (Piaget, 1954, pp. 212). Vista spatial environments refer to Montello's vista space, cf. (Montello, 1993), that is "projectively as large as the human body and that can be apprehended from one place without necessary locomotion". Typical vista spaces are single rooms, offices, small valleys, etc.

Vista spatial environments are dynamic. For example, chairs and books are often placed here and there in your office, your home. However, you can recognize your office, your home.

Intuitively, we recognize a place not by checking everything in it. The air in the room, a sheet of paper, an apple and contents in the dustbin do not help to recognize the room. This paper addresses the task of recognizing spatial environments by asking what exists in an environment that makes it be that environment. It proposes formal ontologies for recognizing vista spatial environments by extending the $R C C 8$ theory, cf. (Randell et al., 1992). 


\section{The State of the Art}

Recognizing spatial environments relates to perception, cognition and corresponding linguistic expressions, which are all fiat, cf. (Smith, 2001). It has nothing to do with finger print checking, molecule analysis or DNA testing. Therefore this paper strictly distinguishes fiat ontologies from bona fide ontologies, cf. (Smith and Varzi, 2000).

Rosch, et al. (1976) reported that humans recognize single objects at the basic level category. At this level (of granularity) humans find it easiest to name objects and recognize them the fastest. This introduces the question of the formation of object constancy: If you look at an apple for one minute, how do you know that the apple you saw at the beginning of the minute is the same apple you saw at the end of the minute? Piaget (1954) Marr and Nishihara (1978) and Rock (1983) suggested that information about spatial relations of an object or parts of an object are important in object constancy. This is consistent with the case of a brain impaired patient: Wilson, et al. (1999) reported a woman, LE, who suffered from a rare memory problem. LE cannot retrieve images from her memory, thus, she cannot distinguish two windows whose glasses have different images and she even has difficulty in recognizing her husband's face. What she can retrieve are only contours of objects. On the other hand, she can locate objects. Amazingly, she can recognize spatial environments, such as her home ${ }^{1}$. This shows that recognizing spatial environments does not require much information about single objects but rather the spatial relations among them.

The starting point of this paper is that objects in an environment are recognized at basic level categories and that spatial relations among them are known. This paper presents ontologies for recognizing a vista spatial environment on this basis.

This paper follows the philosophy that dynamic spatial ontology should combine two distinct types of inventory of the entities and relationships in reality: on the one hand, a purely spatial ontology supporting snapshot views of the world at successive instants of time: SNAP; on the other hand, a purely spatiotemporal ontology of change and process: SPAN, cf. (Grenon and Smith, 2004). Recognizing a vista spatial environment relates its two snapshots (one is remembered in the mind, the other is currently perceived) and the spatiotemporal relation between them: can they (or to what extent can they) participate in the same SPAN?

The snapshot of a spatial environment in mind is termed a cognitive map in the literature, cf. (Tolman, 1948; Kuipers, 1978). Structures of cognitive maps concerning environmental spaces and geographical spaces are hierarchical, cf. (Kosslyn et al., 1974; Siegel and White, 1975; Steven and Coupe, 1978; Hirtle and Jonides, 1985; Tversky, 1991). This paper proposes that the structure of the cognitive map of a vista spatial environment is also hierarchical and this hierarchy is revealed in the selection of reference objects in spatial linguistic de-

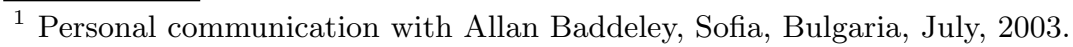


scriptions: a location object is at lower or the same level in the hierarchy as its reference object(s). For example, if people say "the cup is on the table", then in their cognitive map the cup is located lower than or at the same position as the table in the hierarchy. This paper proposes that the level of an object in the hierarchy of a person's cognitive map is determined by commonsense knowledge of its stability in this environment, which is a SPAN ontology.

There is some work on representations of spatial relations among extended objects, cf. (de Laguna, 1922; Randell et al., 1992; Clementini and Di Felice, 1997; Goyal, 2000; Schmidtke, 2001; Schmidtke, 2003). This paper briefly introduces topological definitions of subjective distance and orientation relations between regions for the task of recognizing vista spatial environments.

The paper is structured as follows: Section 3 and section 4 present SNAPVis and SPANVis for recognizing vista spatial environments; section 5 presents the structure of a cognitive map; section 6 briefly outlines the recognition issues; section 7 summarizes the paper.

\section{SNAPVis for Recognizing Vista Spatial Environments}

Following Smith's ontologies of SNAP-SPAN, this article proposes SNAPVis and SPANVis for vista spatial environments. SNAPVis is an ontology for continuants of vista spatial environments; SPANVis is an ontology for occurrents of vista spatial environments.

\subsection{Fiat Regions}

Fiat Boundaries. When we look around, we do not see clusters of atoms or molecules. Rather, we perceive objects and name them at categories. A correspondence between perception and its language description has been proposed by Tversky and Lee (1999) as follows: to the extent that space is schematized similarly in language and cognition, language will be successful in conveying space (the Schematization Similarity Conjecture). Thus, objects recognized through perception are fiat in the sense that they relate to both the objects and human cognition. The fiat boundary of an object refers to its conceptual boundary through perception and the perceptual object is named at the basic level category, such as rooms, windows, doors, furniture, etc. A fiat boundary is an instantiation of a basic level category. Fiat boundaries are named by the object names at basic level categories (may be followed by a natural number to distinguish different instances of the same category), such as "room", "room 1 ", "window and capital lettered names are used as basic level categories, such as "ROOM", "WINDOW". 'BasicLevel $(w)$ ' is a predicate standing for " $w$ is a basic level category"; 'FiatBoundary $(x)$ ' is a predicate which stands for " $x$ is a kind of fiat boundary at the basic level category"; 'Constituent $(x, w)^{2}$ ' is a predicate for " $x$ is an instantiation of the category of $w$ ".

\footnotetext{
${ }^{2}$ This terminology 'Constituent' is adopted from (Grenon and Smith, 2004).
} 
Formulae in this paper have the form of " $(Q x \mid p \bullet q)$ ", $Q$ is the quantifier, $x$ is the bound variable, $p$ is the constraint of $x$, and $q$ is the predicate. It is read as "for all $x$ satisfying $p, q$ holds (or there is $x$ satisfying $p$ such that $q$ )." Any fiat boundary of an extended object belongs to a class.

$$
\forall x \exists w \bullet \text { FiatBoundary }(x) \rightarrow \text { Constituent }(x, w) \wedge \operatorname{BasicLevel}(w)
$$

A fiat boundary is an instantiation of only one basic level category:

$$
\begin{array}{r}
\forall x, w_{1}, w_{2} \mid \text { FiatBoundary }(x) \wedge \text { Constituent }\left(x, w_{1}\right) \wedge \operatorname{BasicLevel}\left(w_{1}\right) \\
\wedge \text { Constituent }\left(x, w_{2}\right) \wedge \operatorname{BasicLevel~}\left(w_{2}\right) \\
\bullet w_{1}=w_{2}
\end{array}
$$

Fiat boundaries of the same basic level category are indistinguishable in isolation. The predicate 'CanBeTheSame $(x, y)$ ' stands for " $x$ and $y$ are indistinguishable in isolation."

$$
\begin{aligned}
& \forall x, y \mid \text { FiatBoundary }(x) \wedge \text { FiatBoundary }(y) \bullet \\
& (\exists w \mid \text { BasicLevel }(w) \bullet \text { Constituent }(x, w) \wedge \text { Constituent }(y, w)) \\
& \quad \rightarrow \text { CanBeTheSame }(x, y)
\end{aligned}
$$

Fiat boundaries of different basic level categories must be different fiat boundaries.

$$
\begin{aligned}
& \forall x, y \mid \text { FiatBoundary }(x) \wedge \text { FiatBoundary }(y) \bullet \\
& \left(\forall w_{1}, w_{2} \mid \text { Constituent }\left(x, w_{1}\right) \wedge \text { Constituent }\left(y, w_{2}\right)\right. \\
& \wedge \operatorname{BasicLevel}\left(w_{1}\right) \wedge \operatorname{BasicLevel}\left(w_{2}\right) \\
& \left.\bullet w_{1} \neq w_{2} \rightarrow \neg \operatorname{CanBeTheSame}(x, y)\right)
\end{aligned}
$$

Fiat Parts. Humans recognize spatial objects by perceiving their partial images, cf. (Buelthoff and Edelman, 1992; Humphrey and Khan, 1992; Tarr, 1995).

'RecognizablePart $(y, x)$ ' stands for " $y$ is a recognizable part of fiat boundary $x$, which can be used to recognize $x$ " ; 'recognition $(y)$ ' is a function which returns the basic level category of a recognizable part $y$.

Recognizable parts of a fiat boundary are used to identify its basic level category:

$$
\begin{aligned}
& \forall x, y, w \mid \text { FiatBoundary }(x) \wedge \text { RecognizablePart }(y, x) \\
& \wedge \operatorname{BasicLevel}(w) \bullet w=\operatorname{recognition}(y) \rightarrow \operatorname{Constituent}(x, w)
\end{aligned}
$$

Recognizable parts of an object are often different. Your face seen from the front is completely different from that seen from the left side, though both are recognizable parts of the face. ' $\operatorname{front}(x)^{\prime}$ ', 'le $f t(x)$ ', $\operatorname{right}(x)$ ' and 'behind $(x)$ ' 

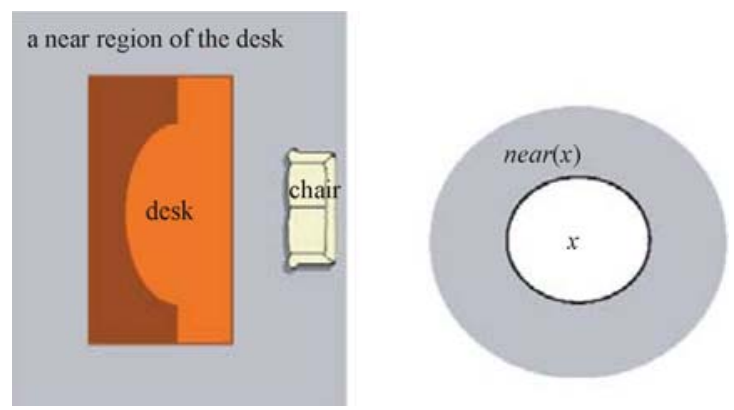

Fig. 1. Fiat extension of spatial region: the near region of the desk

stand for the front, left, right and behind recognizable parts of the fiat boundary $x$, respectively. They are linguistic fiat after (Smith, 2001).

$$
\begin{aligned}
& \forall x, y \mid \text { FiatBoundary }(x) \bullet y=\operatorname{front}(x) \rightarrow \operatorname{RecognizablePart}(y, x) \\
& \forall x, y \mid \text { FiatBoundary }(x) \bullet y=\operatorname{left}(x) \rightarrow \operatorname{RecognizablePart}(y, x) \\
& \forall x, y \mid \text { FiatBoundary }(x) \bullet y=\operatorname{right}(x) \rightarrow \operatorname{RecognizablePart}(y, x) \\
& \forall x, y \mid \text { FiatBoundary }(x) \bullet y=\operatorname{behind}(x) \rightarrow \operatorname{RecognizablePart}(y, x)
\end{aligned}
$$

Fiat Extensions of Spatial Regions. For a description like "the chair is near the writing-desk", it is normally explained that the spatial relation between the chair and the writing-desk is near. This article takes an alternative view as follows: the fiat boundary of the chair is overlapped with the near region of the desk, shown in Figure 1.

The Near Function. If $x$ is a fiat boundary, then near $(x)$ is a fiat region which denotes the nearby region of $x$. The closure of the near $(x)$ region is a ring which has two boundaries: an inner boundary and a fiat outer boundary. The inner boundary coincides with the fiat boundary $x$, as we would not say an object is near to a region when it is already connected with the region. The outer boundary is determined by both the objects and human cognition. So the near $(x)$ region is fiat and its closure is externally connected with the region ${ }^{3} x$. The near function is similar with the 'penumbra region' in (Freksa, 1981) and 'Egg-Yolk' in (Cohn and Gotts, 1996), however, it can be applied recursively. That is, a near region can have its own near region. Formal definitions are given in the appendix, as detailed discussion is beyond the scope of the article.

The near function is only a special case of fiat extensions of fiat boundaries. " $A$ is nearer to $B$ than to $C$ " can be further explained as: there is a fiat extension of $A$ which is connected with the fiat boundary $B$ and is disconnected with the fiat boundary $C$. The function of ' fiat_extension $^{n}(x)$ ' is defined as:

\footnotetext{
${ }^{3}$ The fiat boundary of a 3-dimensional object is a 2-dimensional region.
} 


$$
\text { fiat_extension }^{n}(x) \stackrel{\text { def }}{=} x \cup \text { near }(x) \cup \text { near }^{2}(x) \cup \cdots \cup n \text { near }^{n}(x)
$$

Then:

$$
\begin{aligned}
& \forall x, y, z \mid \text { FiatBoundary }(x) \wedge \text { FiatBoundary }(y) \\
& \wedge \text { FiatBoundary }(z) \bullet \text { nearer }(x, y, z) \stackrel{\text { def }}{=} \\
& \exists n \bullet C\left(\text { fiat_extension }^{n}(x), y\right) \wedge D C\left(\text { fiat_extension }^{n}(x), z\right)
\end{aligned}
$$

where $C$ and $D C$ mean "connected with" and "disconnected with" respectively (following the notions in $R C C$ ).

\subsection{Spatial Relations}

Spatial relations include classic topologic relations, qualitative distance relations and qualitative orientation relations, cf. (Stock, 1997).

Classic Topologic Relations. Classic topological relations depict connectedness relations between two regions, as described by $R C C 8$.

Qualitative Orientation Relations. The different observed recognizable parts result in the orientation relations between the observer and the object. When you are watching television (i.e., you are looking at the recognizable part of the TV set which provides sequences of images), you know that you are in front of the TV set.

Such orientation relations can be formalized by a comparison of qualitative distances: "the chair is in front of the desk" means that the chair is nearer to the front fiat part of the desk than its other fiat parts, shown in Figure 2.

'Front $(x, y)$ ' stands for " $x$ is in front of $y$ or in the front of $y$ ":

$$
\begin{aligned}
& \forall x, y \mid \text { FiatBoundary }(x) \wedge \text { FiatBoundary }(y) \bullet \operatorname{Front}(x, y) \stackrel{\text { def }}{=} \\
& \forall z \mid \operatorname{RecognizablePart}(z, y) \bullet z \neq \operatorname{front}(y) \rightarrow \operatorname{nearer}(x, \operatorname{front}(y), z)
\end{aligned}
$$

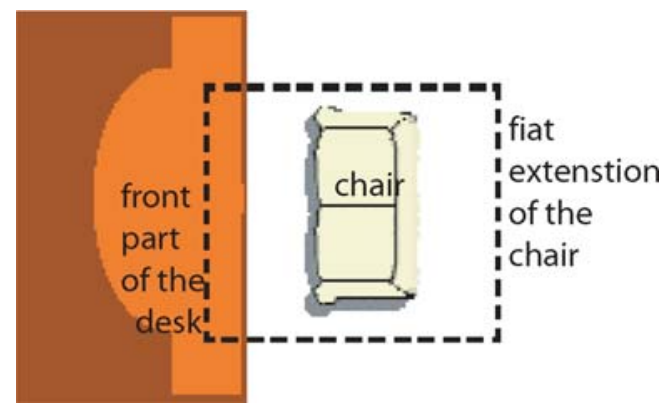

Fig. 2. The qualitative orientation relation is explained by the comparison of qualitative distances among fiat extension region of the location object and fiat parts of the reference object 
Similarly, 'Left $(x, y)$ ' stands for " $x$ is located left of $y$ or left to $y$ "; ' $\operatorname{Right}(x, y)$ ' for " $x$ is located right of $y$ or right to $y$ "; 'Behind $(x, y)$ ' for " $x$ is located behind $y$ or at behind part of $y "$ :

$$
\begin{aligned}
& \forall x, y \mid \text { FiatBoundary }(x) \wedge \text { FiatBoundary }(y) \bullet \operatorname{Left}(x, y) \stackrel{\text { def }}{=} \\
& \forall z \mid \text { RecognizablePart }(z, y) \bullet z \neq \operatorname{left}(y) \rightarrow \operatorname{nearer}(x, \operatorname{left}(y), z) \\
& \forall x, y \mid \text { FiatBoundary }(x) \wedge \text { FiatBoundary }(y) \bullet \operatorname{Right}(x, y) \stackrel{\text { def }}{=} \\
& \forall z \mid \operatorname{RecognizablePart}(z, y) \bullet z \neq \operatorname{right}(y) \rightarrow \operatorname{nearer}(x, \operatorname{right}(y), z) \\
& \forall x, y \mid \text { FiatBoundary }(x) \wedge \text { FiatBoundary }(y) \bullet \operatorname{Behind}(x, y) \stackrel{\text { def }}{=} \\
& \forall z \mid \operatorname{RecognizablePart}(z, y) \bullet z \neq \operatorname{behind}(y) \rightarrow \operatorname{nearer}(x, \operatorname{behind}(y), z)
\end{aligned}
$$

Qualitative Distance Relations. Qualitative distance relations specify the ' $D C$ ' relation in RCC by giving qualitative distance relations such as ' $N R$ '(near), ' $P R$ ' (penumbra far-or-near), and ' $F R$ '(far), as we seldom use an expression like "the chair is disconnected with the desk", but instead we give qualitative distance relations, such as "the chair is near the desk".

Fiat boundary $x$ near fiat boundary $y$ is defined as: $x$ is disconnected with $y$ and overlapped with the near region of $y$ :

$$
\begin{gathered}
\forall x, y \mid \text { FiatBoundary }(x) \wedge \text { FiatBoundary }(y) \bullet \\
N R(x, y) \stackrel{\text { def }}{=} D C(x, y) \wedge O(x, \operatorname{near}(y))
\end{gathered}
$$

Fiat boundary $x$ penumbra far or near $y$ is defined as: $x$ is disconnected with $y$ and externally connected with its near region:

$$
\begin{gathered}
\forall x, y \mid \text { FiatBoundary }(x) \wedge \text { FiatBoundary }(y) \bullet \\
P R(x, y) \stackrel{\text { def }}{=} D C(x, y) \wedge E C(x, \operatorname{near}(y))
\end{gathered}
$$

Fiat boundary $x$ far away from $y$ is defined as: $x$ is disconnected with $y$ and its near region:

$$
\begin{gathered}
\forall x, y \mid \text { FiatBoundary }(x) \wedge \text { FiatBoundary }(y) \bullet \\
F R(x, y) \stackrel{\text { def }}{=} D C(x, y) \wedge D C(x, \operatorname{near}(y))
\end{gathered}
$$

It is obvious that $F R, P R, N R$ are pairwise disjoint. $F R, P R, N R$ also jointly exhausts the $D C$ relation, as shown below:

$$
\begin{gathered}
\forall x, y \mid \text { FiatBoundary }(x) \wedge \text { FiatBoundary }(y) \bullet \\
D C(x, y) \equiv N R(x, y) \vee P R(x, y) \vee F R(x, y)
\end{gathered}
$$

Proof sketch: For all fiat boundaries $x$ and $y$

$$
\begin{aligned}
& N R(x, y) \vee P R(x, y) \vee F R(x, y) \\
& {[(16),(17),(18)]}
\end{aligned}
$$




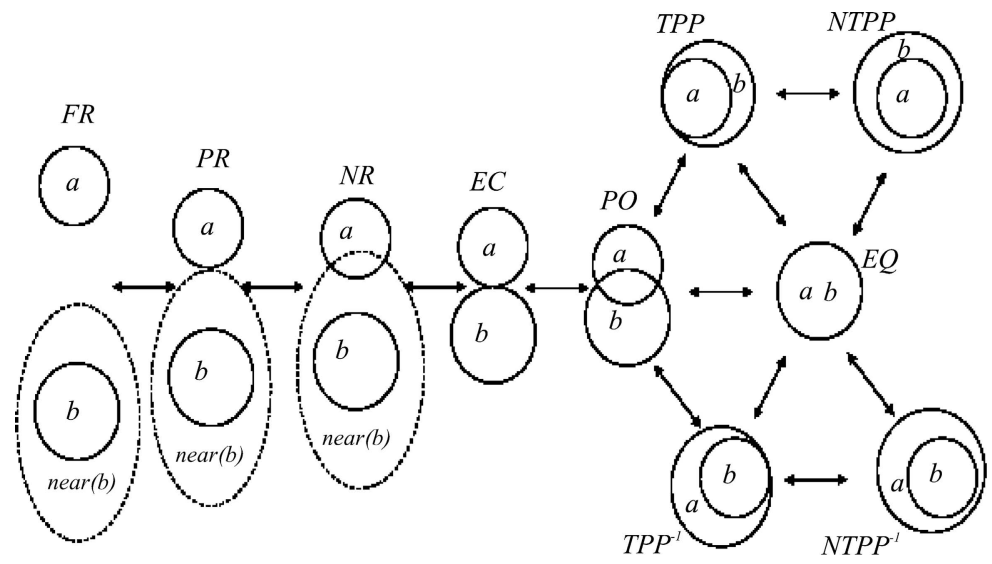

Fig. 3. The ten topological relations $R C C 10$ for vista spatial environments. The disconnected $(D C)$ relation in $R C C 8$ is specified by three qualitative distance relations: far $(F R)$, penumbra far-or-near $(P R)$ and near $(N R)$

$$
\begin{aligned}
\stackrel{\text { def }}{=} D C(x, y) \wedge & O(x, \text { near }(y)) \vee D C(x, y) \wedge E C(x, \text { near }(y)) \\
& \vee D C(x, y) \wedge D C(x, \text { near }(y)) \\
& {[(q \wedge a) \vee(q \wedge b) \vee(q \wedge c) \equiv q \wedge(a \vee b \vee c))] } \\
\equiv & D C(x, y) \wedge(O(x, \text { near }(y)) \vee E C(x, \text { near }(y)) \vee D C(x, \text { near }(y))) \\
& {[\text { Definition of EC and } D C \text { in } R C C] } \\
\equiv & D C(x, y) \wedge(O(x, \text { near }(y)) \vee(C(x, \text { near }(y)) \wedge \neg O(x, \text { near }(y))) \\
& \vee \neg C(x, \text { near }(y))) \\
& {[p \vee(s \wedge t) \equiv(p \vee s) \wedge(p \vee t)] } \\
\equiv & D C(x, y) \wedge(O(x, \text { near }(y)) \vee \neg C(x, \text { near }(y)) \vee C(x, \text { near }(y))) \\
& \wedge(O(x, \text { near }(y)) \vee \neg C(x, \text { near }(y)) \vee \neg O(x, \text { near }(y))) \\
& {[p \vee \neg p \equiv T ; T \vee p \equiv T ; T \wedge T \equiv T ; q \wedge T \equiv q] } \\
\equiv & D C(x, y) \quad
\end{aligned}
$$

This paper therefore proposes a new $\mathbf{R C C 1 0}$ by splitting the $D C$ of $R C C 8$ into three qualitative distance relations for vista spatial recognition. The conceptual neighborhoods network, after the notion in (Freksa, 1991), of RCC10 is shown in Figure 3.

\section{SPANVis for Recognizing Vista Spatial Environments}

\subsection{The Stability}

The stability of a fiat boundary is commonsense knowledge about the spatiotemporal property of the fiat boundary, which affects the selection of reference objects in spatial linguistic descriptions. For example, we neither say "the table is 

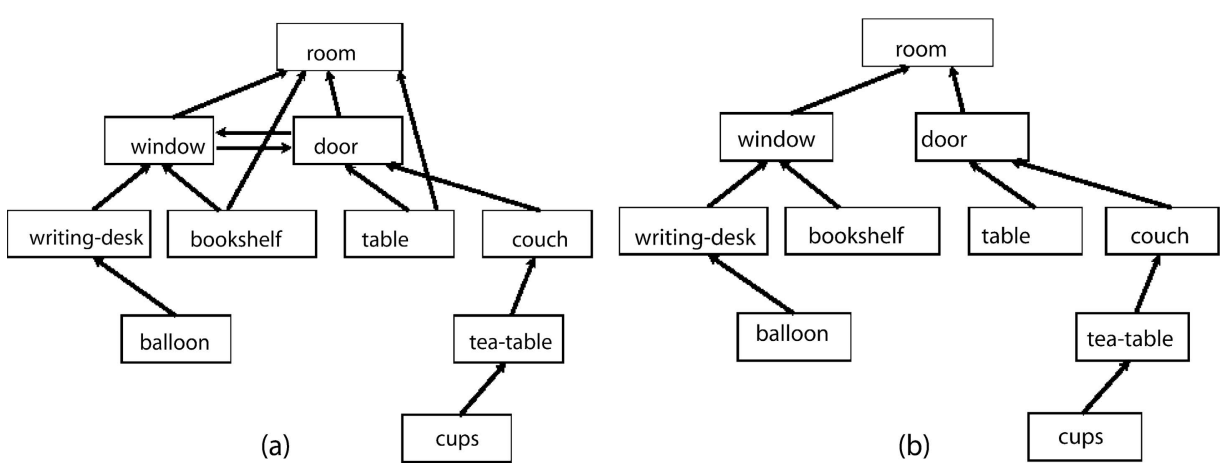

Fig. 4. (a) shows the linguistic reference relations; (b) shows the partial order lattice of direct reference pairs that is generated from (a)

under the book" nor "the wall is behind the picture". Because normally books and pictures are less stable than tables and walls, locating an object by a less stable object provides little information about its location. If a pilot in a plane has lost his current location information, he expects something like "you are above the South Pole" not "you are in your plane". 'stability $(x)$ ' stands for "the stability of fiat boundary $x . "$

\subsection{The Partial Order Lattice of References}

Given a set of spatial linguistic descriptions about a snapshot layout of an environment, the direct reference pair $(A, B)$ is defined as such:

(1) $A$ is used as the reference to $B$ and there is no third object $C$ such that $(A, C)$ and $(C, B)$;

(2) $B$ is not used as the reference to $A$.

All of the direct reference pairs form a partial order lattice. ' $P O L(p l)$ ' standing for " $p l$ is a partial order lattice". I define here for convenience: 'top_level $(p l)$ ', 'second_level(pl)', 'third_level( $p l)$ ' and 'fourth_level( $(p l)$ ' stands for "top level, second level, third level and forth level spatial objects in $p l$, respectively". Objects that are lower than the fourth level cannot help to recognize the room, therefore, they are neglected.

\subsection{An Example}

Given the spatial linguistic descriptions of a remembered scenario, $R S$, as follows: the door is in the wall and opposite to the window; the window is in the wall and opposite to the door; the writing-desk is next to the window; the bookshelf is close to the wall and near the window; the couch is on the left of the door; the balloon is in front of the writing-desk; the tea-table is before the couch, teacups are on the tea-table; the table is near the wall and on the right, if you come through the door, linguistic reference relations are shown in Figure 4(a). Then, its partial order lattice of stability, $p l_{R S}$, can be generated as shown in 
Figure $4(\mathrm{~b})$. top_level $\left(p l_{R S}\right)=\{$ room $\}$, second_level $\left(p l_{R S}\right)=\{$ window,door $\}$, third_level $\left(p l_{R S}\right)=\{$ writingdesk, bookshelf, table, couch $\}$, fourth_level $\left(p l_{R S}\right)$ $=\{$ balloon, teatable $\}$.

\section{The Cognitive Map of Vista Spatial Environments}

To recognize your office, you do not pay attention to the location of apples, books, pens, etc., because they are too un-stable and their locations cannot help you to decide whether it is your target room. Cognitive maps of a vista space only include some of the objects in it.

Foos (1980) investigated constructing cognitive maps from language descriptions. Talmy (1983) discussed how language is effective for conveying spatial information. He proposed that language schematizes space by selecting certain aspects of a referent scene to represent the whole, while disregarding others. The schematization of living spaces in (Ullmer-Ehrich, 1982) discarded all these small objects, like apples, books, pens, etc. and only selected big ones. This suggests that cognitive maps of vista spatial environments only include some objects in the higher levels of the partial order lattice.

A cognitive map of a vista spatial environment, written as ' $\operatorname{Cog} M$ ', is a representation of the subjective knowledge a person has about the environment. It has a hierarchical structure of spatial relations between fiat regions. The hierarchy is formed according to the commonsense knowledge of stability of spatial objects; and objects are anchored to reference objects at the same level or at the neighboring level in the hierarchy. So, it is a partial-hierarchical structure after (McNamara, 1986). A cognitive map represents a set of SNAPVis ontologies partial-hierarchically structured by an implicit SPANVis ontology: the stability. Different persons may have different spatial knowledge; even the same person will use different spatial knowledge for different tasks. When people recognize a vista spatial environment, normally, the first thing they notice is the shape of the environment, then these non-moveable objects, such as doors and windows, if any, and then big furniture ${ }^{4}$. This paper assumes the following structure of a cognitive map: its root is the room; its second level contains windows and doors; its third level contains big furniture like desks, shelves; its fourth level contains chairs, tea-tables, etc. Normally, objects in the top two levels are non-movable; objects in the third level are seldom moved; objects in the fourth level are often moved.

\subsection{The Fiat Container}

The fiat container of an object is a fiat region delineated by a spatial relation and this object. For example, "in front of the desk" "behind the desk" "left of the desk" and "right of the desk" delineate four fiat containers by orientation rela-

${ }^{4}$ Personal communication with Jack Loomis, Bad Zwischenahn, Germany, August, 2003. 
tions and the desk. From the perspective of the fiat container, the spatial relation between the location object and the reference object is interpreted as which fiat container the location object is located in. An object is always located in fiat containers created by upper level or same level objects. 'fiat_container(refO,rel)' or for short ' $\mathfrak{S}_{\text {refo }}(\mathrm{rel})$ ' stands for the fiat container delineated by the reference object refO and the spatial relation rel, which can be one of the $R C C 10$ relations or an orientation relation. ".objects" is an operator of a fiat container which returns the set of objects that are located in this fiat container. For example, fiat_container $\left(\right.$ des $\left._{1}, N R\right)$.objects $=\left\{\operatorname{chair}_{1}\right\}\left(\mathfrak{S}_{\text {des }_{1}}(N R)\right.$.objects $=$ $\left\{\right.$ chair $\left.\left._{1}\right\}\right)$ stands that chair $_{1}$ is located in the near region of desk $k_{1}$; similarly, fiat_container $\left(\right.$ des $_{1}$, Front $)$. objects $=\left\{\right.$ chair $\left._{1}\right\}\left(\mathfrak{S}_{\text {desk }_{1}}(\right.$ Front $)$. objects $=$ $\left\{\right.$ chair $\left.\left._{1}\right\}\right)$ stands that chair $_{1}$ is located in front of or the front part of desk $k_{1}$.

\subsection{Fiat Containers for Locations}

The location of an object refers to the fiat container where the object is located. 'location_distance $(x$, refO $)=\operatorname{rel}_{R C C 10}$ ' is a function that returns a RCC10 relation, which means that $x$ is located in the fiat container created by refO and rel $_{R C C 10}$. E.g., 'location_distance $\left(\right.$ chair $_{1}$, desk $\left._{1}\right)=$ near' means "chair $_{1}$ is located in the near fiat container of desk $k_{1}$ ". 'location_orientation $(x$, refO $)=$ rel $_{O R I}$ ' is a function that returns an orientation relation, which means that $x$ is located in the fiat container created by refO and rel ORI $_{\text {. E.g., 'location_orientation }}$ $\left(\right.$ chair $_{1}$, desk $\left._{1}\right)=$ front' means "chair 1 is located in the front fiat container of desk $_{1} "$.

\subsection{Postures as Orientations}

The posture of an object refers to how it is located, e.g., it faces to the reference object or backs to it. The posture of an object to its reference object can be interpreted as the orientation of the reference object to the object. For example, "the chair faces towards the desk" is interpreted as "the desk is in front of the chair"; if now the chair turns back to the desk, then it is interpreted as "the desk is behind the chair".

\subsection{To Continue the Example}

Suppose the spatial relations in the linguistic descriptions in $\S 4.3$ are formalized in Table 1, then, the fiat containers are:

$$
\begin{aligned}
& \mathfrak{S}_{\text {room }}(P O) . \text { objects }=\{\text { window }, \text { door }\}, \mathfrak{S}_{\text {room }}(T P P) . \text { objects }=\{\text { bookshelf }, \text { table }\} \\
& \mathfrak{S}_{\text {window }}(F R) . \text { objects }=\{\text { door }\}, \mathfrak{S}_{\text {window }}(N R) . \text { objects }=\{\text { writingdesk, bookshelf }\} \\
& \mathfrak{S}_{\text {door }}(F R) . \text { objects }=\{\text { window }\}, \mathfrak{S}_{\text {door }}(\text { Left }) . \text { objects }=\{\text { couch }\} \\
& \mathfrak{S}_{\text {door }}(\text { Right }) . \text { objects }=\{\text { table }\}, \mathfrak{S}_{\text {writingdesk }}(\text { Front }) . \text { objects }=\{\text { balloon }\} \\
& \mathfrak{S}_{\text {couch }}(\text { Front }) . \text { objects }=\{\text { teatable }\}
\end{aligned}
$$


Table 1. Formal representations of spatial relations in the spatial linguistic descriptions

\begin{tabular}{|c|c|c|c|}
\hline descriptions & relation & type & formal relation \\
\hline \multirow{2}{*}{$\begin{array}{l}\text { The door is in the wall } \\
\text { and opposite to the window }\end{array}$} & in & $\mathrm{RCC} 10$ & $P O$ \\
\hline & opposite to & RCC10 & $F R$ \\
\hline \multirow{2}{*}{$\begin{array}{l}\text { the window is in the wall } \\
\text { and opposite to the door }\end{array}$} & in & $\mathrm{RCC} 10$ & $P O$ \\
\hline & opposite to & $\mathrm{RCC} 10$ & $F R$ \\
\hline $\begin{array}{l}\text { the writing-desk is next } \\
\text { to the window }\end{array}$ & next to & RCC10 & $N R$ \\
\hline \multirow{2}{*}{$\begin{array}{l}\text { the bookshelf is close to the wall } \\
\text { and near the window }\end{array}$} & close to & RCC10 & $T P P$ \\
\hline & near & $\mathrm{RCC} 10$ & $N R$ \\
\hline $\begin{array}{l}\text { the couch is on the left } \\
\text { of the door }\end{array}$ & on the left & ORIENTATION & Left \\
\hline $\begin{array}{l}\text { the balloon is in front of } \\
\text { the writing-desk }\end{array}$ & in front of & ORIENTATION & Front \\
\hline \multirow{2}{*}{$\begin{array}{l}\text { the table is near the wall } \\
\text { and on the right, if you come } \\
\text { through the door }\end{array}$} & near & RCC10 & $T P P$ \\
\hline & on the right & ORIENTATION & Right \\
\hline $\begin{array}{l}\text { the tea-table is before } \\
\text { the couch }\end{array}$ & before & ORIENTATION & Front \\
\hline
\end{tabular}

\section{The Spatial Oriented Recognition Process}

Generally speaking, the SPAN structure of a spatial environment is indeterminate in the sense that the movements of spatial objects inside are unpredictable. The exact traces of cars and people on the streets are unpredictable at a given time; the exact locations of books, flowers are unpredictable on the next day. Recognizing spatial environments is not a problem of whether the perceived SNAP participates in the expected SPAN of the environment, rather a problem of whether two SNAPs can participate in the same SPAN of the environment. One relates to the spatial environment before the eyes; the other is the one in the memory.

Considering the variability of vista spatial environments, the recognition problem is refined as follows: recognizing a vista spatial environment means that the perceived vista spatial environment is compatible to the one in mind; which means that the layout of the perceived environment can be easily changed into the layout of the one in mind.

Relating to different stabilities (correspond to different levels in the hierarchy) of fiat boundaries, their easiness of spatial changes differs accordingly. For example, spatial changes of a room are extremely difficult or impossible; location changes of windows or doors are also extremely difficult and in most cases impossible; location changes of desks or shelves are difficult, though possible; location changes of chairs or sitting balls are easy; location changes of books are so easy that they do not help to recognize the spatial environment.

Two $\operatorname{Cog} M$ s are not compatible, when there are spatial differences between objects in the top two levels in hierarchy; two CogMs might be compatible, 
when there are no spatial differences between objects in the top two levels and there are spatial differences between objects in the third level; two $\operatorname{Cog} M \mathrm{~s}$ are compatible, when there are no spatial differences between objects in the top three levels and there are spatial differences between objects in the fourth level; two $\operatorname{Cog} M$ s are very compatible, when there are no spatial differences in the top four levels.

\subsection{The Qualitative Creation or Destruction}

Following (Grenon and Smith, 2004) there are fiat boundaries in the perceived $\operatorname{Cog} M$ that are not in the memorized $\operatorname{Cog} M$, namely qualitative creation, such as a new chair, new flowers, etc. There are fiat boundaries in the memorized $\operatorname{Cog} M$ but not in the perceived $\operatorname{Cog} M$, namely qualitative destruction, such as chairs are moved out, flowers withered and thrown away. Comparison of fiat boundaries is at the basic category level, which means that fiat boundaries of the same basic level category are indistinguishable in isolation, though they are made of different atoms and that entities of different basic level category are distinguishable.

A set of fiat boundaries $F$ determines a set of basic level categories $B_{F}$ such that for each element $b \in B_{F}$ there is $f \in F$ such that Constituent $(f, b)$ and for each element $f \in F$ its basic level category is in $B_{F}$. The sets $F$ and $B_{F}$ further determine the characteristic category set $B C_{F}$ by replacing each element $b \in B_{F}$ with the pair $\left(b, n_{b}\right)$, where $n_{b}$ is the number of elements in $F$ which are of the basic level category $b$. For example, $F=\left\{\right.$ chair $_{1}$, chair $_{2}$, chair $_{3}$, desk $_{1}$, desk $\left._{2}\right\}$, then $B_{F}=\{C H A I R, D E S K\}, B C_{F}=\{(C H A I R, 3),(D E S K, 2)\}$.

' $=$ blc' stands for the basic level equal between two sets of fiat boundaries. Let $F_{1}, F_{2}$ be two sets of fiat boundaries, they are at basic level equal, if and only if their characteristic category sets are equal. That is, $F_{1}={ }_{b l c} F_{2}$ iff $B C_{F_{1}}=B C_{F_{2}}$.

' $\ominus$ ' stands for the basic level minus between one set and one element. Let $F$ be a set of fiat boundaries: $F=\left\{f_{1}, f_{2}, f_{3}, \cdots, f_{n}\right\}$ and $f^{\prime} \notin F$.

$$
\begin{aligned}
& F \ominus f^{\prime}= \\
& \iota F_{\text {result }}(\exists f, w \mid f \in F \wedge \operatorname{BasicLevel}(w) \wedge \text { Constituent }(f, w) \\
& \left.\quad \wedge \text { Constituent }\left(f^{\prime}, w\right) \bullet F_{\text {result }}=F-\{f\}\right)
\end{aligned}
$$

' $\iota y(p)$ ': ' $\iota$ ' is the descriptor, ' $\iota y(p)$ ' means such $y$ that satisfies $p$. Though $F \ominus f^{\prime}$ may result in different set of fiat boundaries, they have a unique characteristic category set.

The basic level minus between two sets $F$ and $F^{\prime}$ is defined as:

$$
\begin{aligned}
F \ominus \emptyset & =F \\
F \ominus F^{\prime} & =\iota F_{\text {result }}\left(\exists f \in F^{\prime} \bullet F_{\text {result }}=(F \ominus f) \ominus\left(F^{\prime}-\{f\}\right)\right)
\end{aligned}
$$

'AppearOrDisappear $\left(F_{1}, F_{2}\right)$ ' stands for 'qualitative creations and destructions between $F_{1}$ and $F_{2} "$.

$$
\text { AppearOrDisappear }\left(F_{1}, F_{2}\right)=F_{1} \ominus F_{2} \cup F_{2} \ominus F_{1}
$$




\subsection{Qualitative Spatial Changes}

There are fiat boundaries in the perceived $\operatorname{Cog} M$ that are located differently in the memorized $\operatorname{Cog} M$, such as chairs are moved from near the desk to near the shelf. As fiat boundaries are located in fiat containers created by reference objects in the upper level or the same level, finding location change is pursued by checking whether there are qualitative creations or destructions in fiat containers created by peer objects with the same spatial relation.

Two fiat containers are peer fiat containers, if and only if the two reference objects are of the same basic level category and the two relations are the same.

Suppose that fiat containers shown in $\S 5.4$ are of the remembered environment and that fiat containers of the current perceived environment are as follows:

$$
\begin{aligned}
& \mathfrak{S}_{\text {room }_{2}}(P O) \text {.objects }=\left\{\text { window }_{2}, \text { door }_{2}\right\} \\
& \mathfrak{S}_{\text {room }_{2}}(T P P) . \text { objects }=\left\{\text { bookshel } f_{2}, \text { table }_{2}\right\}, \mathfrak{S}_{\text {window }_{2}}(F R) . \text { objects }=\left\{\text { door }_{2}\right\} \\
& \mathfrak{S}_{\text {window }_{2}}(N R) \text {.objects }=\left\{\text { writingdesk }_{2}, \text { bookshel } f_{2}\right\} \\
& \mathfrak{S}_{\text {door }_{2}}(F R) . \text { objects }=\left\{\text { window }_{2}\right\}, \mathfrak{S}_{\text {door }_{2}}(\text { Right }) . \text { objects }=\left\{\text { table }_{2}, \text { couch }_{2}\right\} \\
& \mathfrak{S}_{\text {door }_{2}}(\text { Left }) . \text { objects }=\left\{\text { couch }_{2}\right\}, \mathfrak{S}_{\text {couch }_{2}}(\text { Front }) . \text { objects }=\left\{\text { teatable }_{2}, \text { balloon }_{2}\right\}
\end{aligned}
$$

Then, peer fiat containers are:

$\mathfrak{S}_{\text {room }}(P O)$ and $\mathfrak{S}_{\text {room }}(P O), \mathfrak{S}_{\text {room }}(T P P)$ and $\mathfrak{S}_{\text {room }}(T P P)$, $\mathfrak{S}_{\text {window }}(F R)$ and $\mathfrak{S}_{\text {window }}(F R), \mathfrak{S}_{\text {window }}(N R)$ and $\mathfrak{S}_{\text {window }}(N R)$, $\mathfrak{S}_{\text {door }}(F R)$ and $\mathfrak{S}_{\text {door }}(F R), \mathfrak{S}_{\text {door }}($ Right $)$ and $\mathfrak{S}_{\text {door }}($ Right $)$, $\mathfrak{S}_{\text {door }}($ Left $)$ and $\mathfrak{S}_{\text {door }}($ Left $), \mathfrak{S}_{\text {couch }}($ Front $)$ and $\mathfrak{S}_{\text {couch }}($ Front $)$

'ExistingSpatialChange $\left(\operatorname{Cog} M_{1}, \operatorname{Cog} M_{2}\right)$ ' stands for 'there are qualitative creations or destructions between the two cognitive maps $\operatorname{Cog} M_{1}$ and $\operatorname{Cog} M_{2}$. It returns the union of the object sets of all the qualitative creations or destructions of their peer fiat containers and non-peered fiat containers in $\operatorname{Cog} M_{1}$ and $\operatorname{Cog} M_{2}$.

For example, let $\operatorname{Cog} M_{R S}$ be the cognitive map of the remembered scenario whose partial order lattice is shown in $\S 4.3$ and fiat containers are shown in $\S 5.4$, $\operatorname{Cog} M_{P S}$ be the cognitive map of the perceived scenario whose fiat containers are shown above, then their spatial differences are observed as follows:

$$
\begin{aligned}
& \text { ExistingSpatialChange }\left(\operatorname{Cog} M_{R S}, \operatorname{Cog} M_{P S}\right) \\
& =\text { AppearOrDisappear }\left(\mathfrak{S}_{\text {room }}(P O) \text {.objects, } \mathfrak{S}_{\text {room }}(P O) \text {.objects }\right) \\
& \cup \text { AppearOrDisappear }\left(\mathfrak{S}_{\text {room }}(T P P) \text {.objects, } \mathfrak{S}_{\text {room }}(T P P) \text {.objects }\right) \\
& \cup \text { AppearOrDisappear }\left(\mathfrak{S}_{\text {window }}(F R) \text {.objects, } \mathfrak{S}_{\text {window }_{2}}(F R) . \text { objects }\right) \\
& \cup \text { AppearOrDisappear }\left(\mathfrak{S}_{\text {window }}(N R) \text {.objects, } \mathfrak{S}_{\text {window }}(N R) . \text { objects }\right) \\
& \cup \text { AppearOrDisappear }\left(\mathfrak{S}_{\text {door }}(F R) \text {.objects, } \mathfrak{S}_{\text {door }_{2}}(F R) \text {.objects }\right) \\
& \cup \text { AppearOrDisappear }\left(\mathfrak{S}_{\text {door }}(\text { Right }) . \text { objects, } \mathfrak{S}_{\text {door }_{2}}\right. \text { (Right).objects) } \\
& \cup \text { AppearOrDisappear }\left(\mathfrak{S}_{\text {couch }}(\text { Front }) . \text { objects, } \mathfrak{S}_{\text {couch }_{2}}\right. \text { (Front).objects) } \\
& \cup \text { AppearOrDisappear }\left(\mathfrak{S}_{\text {door }}(\text { Left }) . \text { objects, } \mathfrak{S}_{\text {door }_{2}}(\text { Left }) . \text { objects }\right) \\
& \cup \mathfrak{S}_{\text {writingdesk }}(\text { Front).objects } \\
& =\{\text { window } \text { door }\} \ominus\left\{\text { window }_{2}, \text { door }_{2}\right\} \cup\left\{\text { window }_{2}, \text { door }_{2}\right\} \ominus\{\text { window } \text { door }\}
\end{aligned}
$$




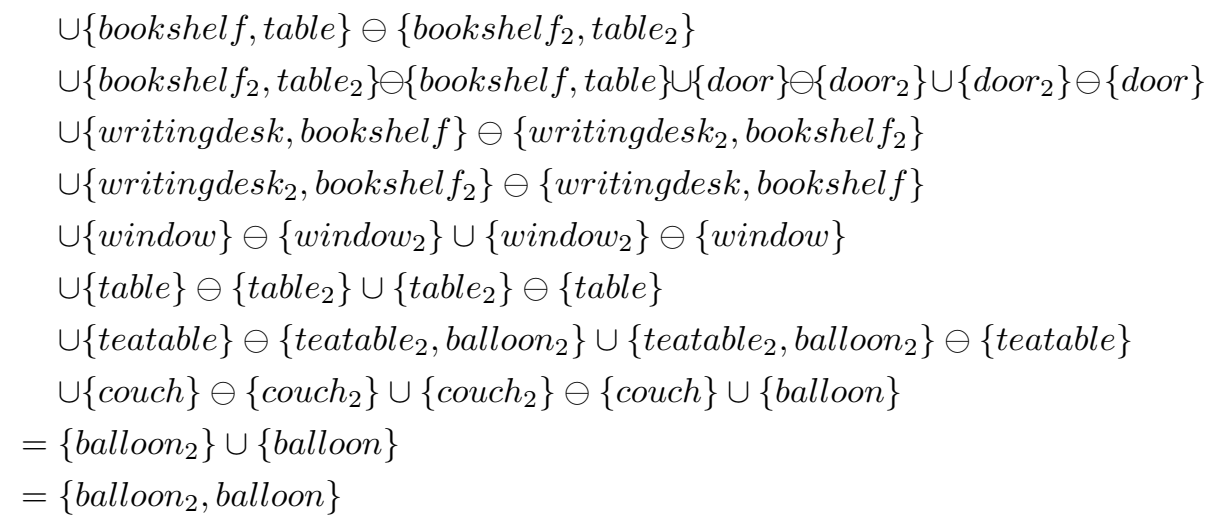

\subsection{The Process of Recognizing Spatial Environments}

The recognition process is active and top-down. Active means that when we go into a vista spatial environment, we have an anticipation of which vista spatial environment it should be. Thus, we have the SNAPVis and the SPANVis of the expected environment in mind, represented by $\operatorname{Cog} M_{r e m}$. Top-down means that spatial objects in the perceived SNAPVis are checked from the top level downwards in the hierarchy.

Let $\operatorname{Cog} M_{\text {rem }}$ and $\operatorname{Cog} M_{\text {per }}$ be the cognitive map remembered and the cognitive map perceived, $p l_{\text {rem }}$ and $p l_{\text {per }}$ be the partial order lattices of the two cognitive maps, respectively.

The perceived $\operatorname{Cog} M$ cannot be compatible with the remembered, if there are any spatial differences of non-movable objects. These objects are located at the top level or the second level in the stability hierarchies.

$$
\begin{aligned}
& \text { NotCompatible }\left(\operatorname{Cog} M_{\text {rem }}, \operatorname{Cog} M_{\text {per }}\right) \stackrel{\text { def }}{=} \\
& \left(\text { ExistingSpatialChanges }\left(\operatorname{Cog}_{\text {rem }}, \operatorname{Cog} M_{\text {per }}\right)\right. \\
& \left.\cap\left(\left(\text { top_level pl } l_{\text {rem }}\right) \cup\left(\text { second_level pl } l_{\text {rem }}\right)\right)\right) \neq \emptyset \\
& \vee\left(\text { ExistingSpatialChanges }\left(\text { Cog } M_{\text {rem }}, \text { Cog } M_{\text {per }}\right)\right. \\
& \left.\quad \cap\left(\left(\text { top_level pl } l_{\text {per }}\right) \cup\left(\text { second_level } p l_{\text {per }}\right)\right)\right) \neq \emptyset
\end{aligned}
$$

The perceived $\operatorname{Cog} M$ might be compatible with the remembered, if there are no spatial differences of non-movable objects and there are spatial differences among big furniture, which are at the third level in the hierarchies.

$$
\begin{aligned}
& \text { MightCompatible }\left(\operatorname{Cog} M_{r e m}, \operatorname{Cog} M_{\text {per }}\right) \stackrel{\text { def }}{=} \\
& \neg \text { NotCompatible }\left(\operatorname{Cog} M_{r e m}, \operatorname{Cog} M_{\text {per }}\right) \\
& \wedge\left(\text { ExistingSpatialChanges }\left(\operatorname{Cog} M_{r e m}, \operatorname{Cog} M_{p e r}\right)\right. \\
& \left.\quad \cap\left(\left(\text { third_level } p l_{r e m}\right) \cup\left(\text { third_level } p l_{\text {per }}\right)\right)\right) \neq \emptyset
\end{aligned}
$$

The perceived $\operatorname{Cog} M$ are compatible with the remembered, if there are neither spatial differences of non-movable objects nor spatial differences of big fur- 
niture and there are spatial differences of small pieces of furniture which are at the fourth level in the hierarchies.

$$
\begin{aligned}
& \text { Compatible }\left(\operatorname{Cog} M_{\text {rem }}, \operatorname{Cog} M_{\text {per }}\right) \stackrel{\text { def }}{=} \\
& \neg \text { NotCompatible }\left(\operatorname{Cog} M_{\text {rem }}, \operatorname{Cog} M_{\text {rem }}\right) \\
& \wedge \neg \text { MightCompatible }\left(\operatorname{Cog} M_{\text {rem }}, \operatorname{Cog} M_{\text {per }}\right) \\
& \wedge\left(\text { ExistingSpatialChanges }\left(\operatorname{Cog} M_{\text {rem }}, \operatorname{Cog} M_{\text {per }}\right)\right. \\
& \left.\quad \cap\left(\left(\text { fourth_level } p l_{\text {rem }}\right) \cup\left(\text { fourth_level } p l_{\text {per }}\right)\right)\right) \neq \emptyset
\end{aligned}
$$

The perceived SNAPVis are very compatible with the remembered, if there is no spatial differences in the top four levels.

$$
\begin{aligned}
& \text { VeryCompatible }\left(\operatorname{Cog} M_{\text {rem }}, \operatorname{Cog} M_{\text {per }}\right) \stackrel{\text { def }}{=} \\
& \neg \text { NotCompatible }\left(\operatorname{Cog} M_{r e m}, \operatorname{Cog} M_{\text {per }}\right) \\
& \wedge \neg \text { MightCompatible }\left(\operatorname{Cog} M_{\text {rem }}, \operatorname{Cog} M_{\text {per }}\right) \\
& \wedge \neg \text { Compatible }\left(\operatorname{Cog} M_{\text {rem }}, \operatorname{Cog} M_{\text {per }}\right) \\
& \wedge\left(\text { ExistingSpatialChanges }\left(\operatorname{Cog} M_{\text {rem }}, \operatorname{Cog} M_{p e r}\right)\right. \\
& \left.\quad \cap\left(\left(\text { fourth_level pl } l_{r e m}\right) \cup\left(\text { fourth_level pl } l_{\text {per }}\right)\right)\right)=\emptyset
\end{aligned}
$$

Suppose the top four levels of partial order lattice of $\operatorname{Cog} M_{P S}$ in $\S 6.2$ are as follows: top_level $\left(p l_{P S}\right)=\left\{\operatorname{room}_{2}\right\}$, second_level $\left(p_{P S}\right)=\left\{\right.$ window $_{2}$, door $\left._{2}\right\}$, third_level $\left(p l_{P S}\right)=\left\{\right.$ writingdes $_{2}$, bookshel $f_{2}$, table $_{2}$, couch $\left._{2}\right\}$, fourth_level $\left(p_{P S}\right)=\left\{\right.$ balloon $_{2}$, teatable 2$\}$, then the perceived scenario is compatible with the remembered one:

$$
\begin{aligned}
& \text { NotCompatible }\left(\operatorname{Cog} M_{R S}, \operatorname{Cog} M_{P S}\right) \\
& \stackrel{\text { def }}{=} \text { (ExistingSpatialChanges }\left(\operatorname{Cog} M_{R S}, \operatorname{Cog} M_{P S}\right) \\
& \left.\cap\left(\left(\text { top_level } p l_{R S}\right) \cup\left(\text { second_level } p l_{R S}\right)\right)\right) \neq \emptyset \\
& \vee\left(\text { ExistingSpatialChanges }\left(\operatorname{Cog} M_{R S}, \operatorname{Cog} M_{P S}\right)\right. \\
& \left.\cap\left(\left(\text { top_level } p l_{P S}\right) \cup\left(\text { second_level } p l_{P S}\right)\right)\right) \neq \emptyset \\
& \equiv\left\{\text { balloon }_{2} \text {, balloon }\right\} \cap(\{\text { room }\} \cup\{\text { window, door }\}) \neq \emptyset \\
& \vee\left\{\text { balloon }_{2}, \text { balloon } \cap\left(\left\{\text { room }_{2}\right\} \cup\left\{\text { window }_{2}, \text { door }_{2}\right\}\right) \neq \emptyset\right. \\
& \equiv F \\
& \text { MightCompatible }\left(\operatorname{Cog} M_{R S}, \operatorname{Cog} M_{P S}\right) \\
& \stackrel{\text { def }}{=} \neg N \text { otCompatible }\left(\operatorname{Cog} M_{R S}, \operatorname{Cog} M_{P S}\right) \\
& \wedge\left(\text { ExistingSpatialChanges }\left(\operatorname{Cog} M_{R S}, \operatorname{Cog} M_{P S}\right)\right. \\
& \left.\cap\left(\left(\text { third_level } p l_{R S}\right) \cup\left(\text { third_level } p l_{P S}\right)\right)\right) \neq \emptyset \\
& \equiv \neg F \wedge\left\{\text { balloon }_{2}, \text { balloon }\right\} \cap(\{\text { writingdesk, bookshelf }, \text { table }, \text { couch }\} \\
& \left.\cup\left\{\text { writingdes }_{2} \text {, bookshel } f_{2}, \text { table }_{2}, \text { couch }_{2}\right\}\right) \neq \emptyset \\
& \equiv F
\end{aligned}
$$




$$
\begin{aligned}
& \text { Compatible }\left(\operatorname{Cog} M_{R S}, \operatorname{Cog} M_{P S}\right) \\
& \stackrel{\text { def }}{=} \neg N \text { otCompatible }\left(\operatorname{Cog} M_{R S}, \operatorname{Cog} M_{P S}\right) \\
& \wedge \neg \text { MightCompatible }\left(\operatorname{Cog} M_{R S}, \operatorname{Cog} M_{P S}\right) \\
& \wedge\left(\text { ExistingSpatialChanges }\left(\operatorname{Cog} M_{R S}, \operatorname{Cog} M_{P S}\right)\right. \\
& \left.\cap\left(\left(\text { fourth_level } p l_{R S}\right) \cup\left(\text { fourth_level } p l_{P S}\right)\right)\right) \neq \emptyset \\
& \equiv \neg F \wedge \neg F \wedge\left\{\text { balloon }_{2} \text {, balloon }\right\} \cap(\{\text { balloon, teatable }\} \\
& \left.\cup\left\{\text { balloon }_{2}, \text { teatable }_{2}\right\}\right) \neq \emptyset \\
& \equiv T
\end{aligned}
$$

A symbolic simulation system, the LIVE model, has been successfully implemented in Lisp. One example is shown in Figure 5.

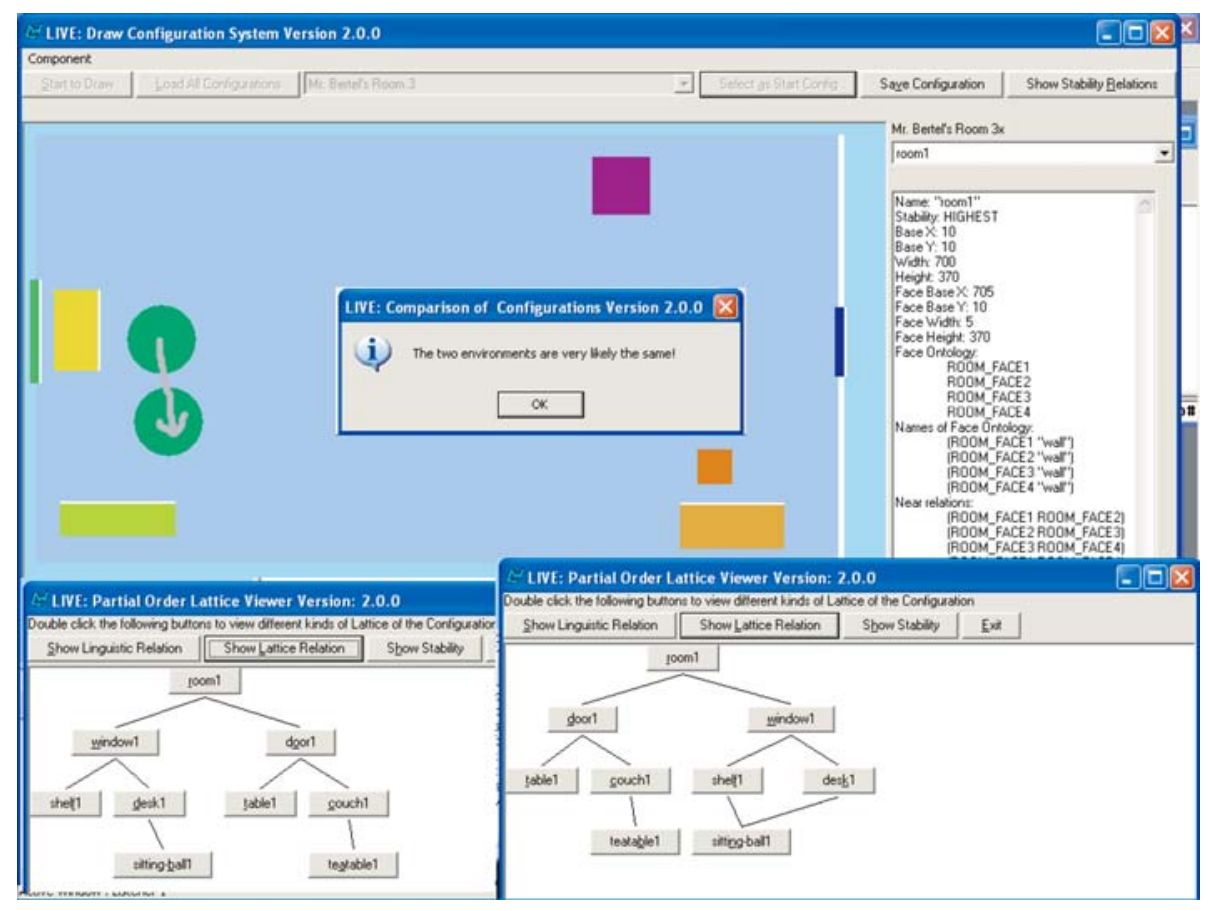

Fig. 5. The sitting-ball in Mr. Bertel's room has been changed a little; its partial order lattice changes accordingly. The recognition process shows that it is Mr. Bertel's room notwithstanding. "to be very likely the same" means: to be the same in most cases

\section{Conclusions}

This article presents basic formal ontologies for recognizing vista spatial environments. The basic topic in vista spatial cognition is how to define spatial relations between extended objects. Qualitative distance relations are defined as 
the "connectedness" relations between the location object and the near region of the reference object; qualitative orientation relations are defined as the "connectedness" relations between the location object and the fiat parts of the reference object. The SNAPVis ontologies are, therefore, constructed by regions occupied by extended objects and the connectedness relations between fiat regions determined by extended objects and human cognition. The stability (a SPANVis ontology) is introduced through the observation of the asymmetry between the location object and its reference object in spatial linguistic descriptions. The basic task of vista spatial cognition is how to recognize variable vista environments. This article proposes that recognizing vista spatial environments is the problem of the compatibility between the remembered environment and the perceived environment, which is determined by spatial differences and stabilities of related objects.

\section{Acknowledgements}

This work has been carried out in the framework of the SFB/TR 8 Spatial Cognition. Financial supports by DAAD IQN (International Quality Network) scholarship and DAAD research scholarship are greatly acknowledged. I am indebted to Christian Freksa for his supervision, to Diedrich Wolter for his early comments that helped me to shape the concept of the near extension region presented in the appendix, to John Bateman for critiques, comments and language improvements on the earlier drafts of this paper, to Barry Smith, Hardwig Hochmair, Jan Oliver Wallgrün, Till Mossakowski, Sven Bertel, Shou-Ren Lu and Christian Freksa for comments on the later drafts, and to three anonymous reviewers for their critiques, comments and supports.

\section{References}

Buelthoff, H. H. and Edelman, S. (1992). Psychophysical support for a two-dimensional view interpolation theory of object recognition. In Proceedings of National Academy of Science, pages 60-64. USA.

Clementini, E. and Di Felice, P. (1997). Approximate topological relations. International Journal of Approximate Reasoning, 16:173-204.

Cohn, A. G. and Gotts, N. M. (1996). The 'egg-yolk' representation of regions with indeterminate boundaries. In Burrough, P. and Frank, A. U., editors, Proceedings, GISDATA Specialist Meeting on Geographical Objects with Undetermined Boundaries, pages 171-187. Francis \& Taylor.

de Laguna, T. (1922). Point, line and surface as sets of solids. The Journal of Philosophy, 19:449-461.

Foos, P. W. (1980). Constructing Cognitive Maps From Sentences. Journal of Experimental Psychology: Human Learning and Memory, 6(1):25-38.

Freksa, C. (1981). Linguistic Pattern Characterization and Analysis. PhD thesis, Department of Electrical Engineering and Computer Science, University of California, Berkeley. 
Freksa, C. (1991). Conceptual Neighborhood and its role in temporal and spatial reasoning. In Singh, M. and Trav-Massuys, L., editors, Decision Support Systems and Qualitative Reasoning, pages 181-187. Elsevier Science Publishers, North-Holland.

Goyal, R. (2000). Similarity Assessment for Cardinal Directions between Extended Spatial Objects. PhD thesis, Spatial Information Science and Engineering, University of Maine.

Grenon, P. and Smith, B. (2004). SNAP and SPAN: Towards Dynamic Spatial Ontology. Spatial Cognition and Computation, 4(1):69-103. Lawrence Erlbaum Associates, Inc.

Hirtle, S. C. and Jonides, J. (1985). Evidence of hierarchies in cognitive maps. Memory \& Cognition, 13(3):208-217.

Humphrey, G. K. and Khan, S. C. (1992). Recognizing novel views of three-dimensional objects. Canadian Journal of Psychology, 46:170-190.

Kosslyn, S. M., Pick, H. L., and Fariello, G. R. (1974). Cognitive maps in children and men. Child Development, 45:707-716.

Kuipers, B. (1978). Modeling spatial knowledge. Cognitive Science, pages 129-153.

Marr, D. and Nishihara, H. K. (1978). Representation and recognition of the spatial organization of three dimensional shapes. In Proceedings of the Royal Society of London, pages 269-294. B 200.

McNamara, T. P. (1986). Mental Representation of Spatial Relations. Cognitive Psychology, 18:87-121.

Montello, D. (1993). Scale and Multiple Psychologies of Space. In Frank, A. and I.Campari, editors, Spatial information theory: A theoretical basis for GIS, pages 312-321. Springer, Berlin.

Piaget, J. (1954). The Construction of Reality in the Child. Routledge \& Kegan Paul Ltd.

Randell, D., Cui, Z., and Cohn, A. (1992). A spatial logic based on regions and connection. In Nebel, B., Swartout, W., and Rich, C., editors, Proc. 3rd Int. Conf. on Knowledge Representation and Reasoning, pages 165-176, San Mateo. Morgan Kaufmann.

Rock, I. (1983). The logic of perception. MIT Press, Cambridge, MA.

Rosch, E., Mervis, C. B., Gray, W., Johnson, D., and Boyes-Braem, P. (1976). Basic objects in natural categories. Cognitive Psychology, 8:382-439.

Schmidtke, H. (2001). The House Is North of the River: Relative Localization of Extended Objects. In Montello, D. R., editor, COSIT 2001, pages 415-430. SpringerVerlag.

Schmidtke, H. (2003). A Geometry for Places: Representing Extension and Extended Objects. In Kuhn, W., Worboys, M. F., and Timpf, S., editors, COSIT 2003, pages 221-238. Springer-Verlag.

Siegel, A. W. and White, S. H. (1975). The development of spatial representation of large-scale environments. In Reese, H., editor, Advances in child development and behaviour, pages 9-55. Academic Press, San Diego, CA.

Smith, B. (2001). Fiat objects. Topoi, 20(2):131-148.

Smith, B. and Varzi, A. C. (2000). Fiat and bona fide boundaries. Philosophy and Phenomenological Research, 60(2):401-420.

Steven, A. and Coupe, P. (1978). Distance estimation from cognitive maps. Cognitive Psychology, 13:526-550.

Stock, O., editor (1997). Spatial and Temporal Reasoning. Kluwer Academic Publishers.

Talmy, L. (1983). How Language Structures Space. In Pick, H. and Acredolo, L., editors, Spatial Orientation: Theory, Research and Application, pages 225-281. Plenum Press. 
Tarr, M. J. (1995). Rotating objects to recognize them: A case study of the role of mental transformations in the recognition of three-dimensional objects. Psychonomic Bulletin and Review, 2:55-82.

Tolman, E. C. (1948). Cognitive Maps in Rats and Men. The Psychological Review, $55(4): 189-208$.

Tversky, B. (1991). Spatial Mental Models. The Psychology of Learning and Motivation, 27:109-145.

Tversky, B. and Lee, P. (1999). How space structures language. In Freksa, C., Habel, C., and Wender, K. F., editors, Spatial Cognition, volume 1404 of LNAI, pages 157-176. Springer-Verlag.

Ullmer-Ehrich, V. (1982). The Structure of Living Space Descriptions. In Jarvella, R. J. and Klein, W., editors, Speech, Place, and Action, pages 219-249. John Wiley \& Sons Ltd.

Wilson, B., Baddeley, A., and Young, A. (1999). LE, A Person Who Lost Her 'Mind's Eye'. Neurocase, 5:119-127.

\section{A Formal Definitions}

This appendix presents simple descriptions of three basic terminologies: the near extension, the near region, and the extension region.

Regions are denoted by mathcal capital letters, such as $\mathcal{A}, \mathcal{B}, \mathcal{C}, \ldots$ Regions belong to classes and the classes of regions are denoted by typewriter capital letters, such as A, B, C, ...

\section{A.1 Primitives and Postulates}

The 'Region' is Primitive. A "region" refers to the space that is occupied by an extended object ${ }^{5}$. This is not a definition of regions, rather how it is understood. A "region" has the following properties, which are used as postulates.

The Postulate of Categories. Humans are preferable to recognize single objects at the basic level category. Accordingly, a "region" belongs to a class. "CL" stands for the set of all the classes of regions that a person has.

Axiom-CL 1. CL is non-empty.

$$
C L \neq \emptyset
$$

For any recognized object, it belongs to one and only one element in CL.

Axiom-CL 2. Any region belongs to a class which is an element of CL.

$$
\forall \mathcal{A} \exists \mathrm{A} \mid \mathrm{A} \in \mathrm{CL} \bullet \mathcal{A} \in \mathrm{A}
$$

Axiom-CL 3. Any region belongs to only one element in $\mathrm{CL}$.

$$
\forall \mathcal{A}, \mathrm{A}, \mathrm{B} \mid \mathrm{A} \in \mathrm{CL} \wedge \mathrm{B} \in \mathrm{CL} \bullet \mathcal{A} \in \mathrm{A} \wedge \mathcal{A} \in \mathrm{B} \rightarrow \mathrm{A}=\mathrm{B}
$$

\footnotetext{
${ }^{5}$ By "an object", I do not exclude objects, such as a hole, or a niche. Thus, the "region"
} here refers to the space occupied by a conceptual object, rather than substances. 
'Connectedness' is Primitive. The only primitive relation between regions is "connected with": $\mathbf{C}$.

\section{A.2 The Near Extension, the Near Region}

Let $\mathcal{A}$ (of the class $\mathrm{A} \in \mathrm{CL}$ ) and $\mathcal{X}$ (of the class $\mathrm{X} \in \mathrm{CL}$ ) be two regions. The near extension of $\mathcal{A}$ by $\mathcal{X}$, written as $\mathcal{A}^{\mathcal{X}}$, refers to the sum of all regions of the class $\mathrm{X}$ that are connected with the region $\mathcal{A}$. $\mathcal{X}$ is called the extension region.

Formally, the near extension of $\mathcal{A}$ by $\mathcal{X}$ can be defined as the region $\mathcal{Y}$ such that, given any region $\mathcal{W}, \mathcal{W}$ connects with $\mathcal{Y}$ if and only if $\mathcal{W}$ connects with a certain region $\mathcal{V}$ of the class $\mathrm{X}$ such that $\mathcal{V}$ connects $\mathcal{A}$.

$$
\begin{aligned}
& \mathcal{A} \stackrel{\mathcal{X} \text { def }}{=} \\
& \iota \mathcal{Y}(\forall \mathcal{W} \bullet(\mathbf{C}(\mathcal{W}, \mathcal{Y}) \equiv \exists \mathcal{V} \mid \mathcal{V} \in \mathrm{x} \wedge \mathcal{X} \in \mathrm{x} \wedge \mathrm{x} \in \mathrm{CL} \bullet \mathbf{C}(\mathcal{A}, \mathcal{V}) \wedge \mathbf{C}(\mathcal{W}, \mathcal{V})))
\end{aligned}
$$

The near region of $\mathcal{A}$ by $\mathcal{X}$, written as $\mathcal{N}_{\mathcal{A}}^{\mathcal{X}}$, can be defined as the difference of $\mathcal{A}^{\mathcal{X}}$ with $\mathcal{A}: \mathcal{N}_{\mathcal{A}}^{\mathcal{X}}=\operatorname{diff}\left(\mathcal{A}^{\mathcal{X}}, \mathcal{A}\right)$, diff following (Randell et al., 1992).

$\mathcal{A}^{\mathcal{X}}$ and $\mathcal{N}_{\mathcal{A}}^{\mathcal{X}}$ are also regions, therefore, according to Axiom-CL 2 and Axiom-CL 3 each belongs to one and only one class, which is an element of $\mathrm{CL}$. The class of $\mathcal{A}^{\mathcal{X}}$ is written as $\mathrm{A}^{\mathrm{X}}$, the class of $\mathcal{N}_{\mathcal{A}}^{\mathcal{X}}$ is written as $\mathrm{N}_{\mathrm{A}}^{\mathrm{X}}$.

The set $C L$ is, therefore, expanded as follows: if $\mathrm{X} \in \mathrm{CL}$ and $\mathrm{Y} \in \mathrm{CL}$, then $\mathrm{X}^{\mathrm{X}}$, $\mathrm{X}^{\mathrm{Y}}$ and $\mathrm{N}_{\mathrm{Y}}^{\mathrm{X}}$ are also elements of $\mathrm{CL}$. For example, suppose that $\mathrm{BIF} \in \mathrm{CL}$ represents the British imperial foot, then $\mathrm{BIF}^{\mathrm{BIF}}{ }^{\mathrm{BIF}} \in \mathrm{CL}$, which represents the class of the yard, see $\S \mathbf{A . 3}$.

\section{A.3 The Extension Regions}

The near extension of $\mathcal{A}$ by $\mathcal{X}$ depends on the class of $\mathcal{X}$, not on the particular $\mathcal{X}$.

For example, British people select their imperial foot as the extension region (the British imperial foot) to measure distance. The British unit of distance were the yard (a yard equals to three feet), and the fathom (a fathom equals to six feet). The British imperial foot is used as the class whose elements have the same length as the British imperial foot. The old French unit of distance was the Paris feet and the toise (a toise equals to six Paris feet).

Ancient Egyptians used the "Royal Egyptian Cubit" as the extension region, which was equal to "the length of the forearm from the bent elbow to the tip of the extended middle finger plus the width of the palm of the hand of the Pharaoh or King ruling at that time" 6 . When ancient Egyptians said that the width of the door was less than five Cubits, it meant that five connected objects (each has the size of "Royal Egyptian Cubit") can connect the two sides of the door. Therefore, the "Royal Egyptian Cubit" was used as the class whose elements have the same length as the Pharaoh's forearm, rather than the concrete Pharaoh's forearm with extended middle finger.

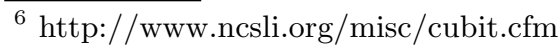


The old German unit of distance ${ }^{7}$ were Elle, the double feet series, a day's journey, etc. The Elle was similar to the "Royal Egyptian Cubit" which was defined as the segment between the bent elbow and the point of extended middle finger; the double feet series used two connected feet as the length-unit, such as Frankfurt double feet, Oldenburg double feet, Bavaria double feet, Vienna double feet, even Hamburg short double feet, Hamburg long double feet, etc.; a day's journey was defined as the distance that can be covered, especially by a horse with cart, in one day ${ }^{8}$. If the horse with cart is replaced with the light and one day is replaced with one year, then we have an extension region in the modern physics - the lightyear that is the distance that light travels in one year.

Ancient Chinese used $D u, C u n, C h i$, etc. as the extension regions. A $D u$ was "two consecutive steps by different legs of a person" ${ }^{9}$. When ancient Chinese measured the length of a road by $D u$, they walked along the road and some of their steps would be inevitably a little bit different from others. $D u$ was, therefore, used as the class of all two consecutive steps of a person. Cun is the body segment between the wrist striation behind the thumb and the pulsing point of the radial artery ${ }^{10} ; C h i$ is the body segment between the wrist striation and the striation at the acupuncture point called "Qu-Chi" in Chinese medicine ${ }^{11}$. Therefore, Cun and Chi are used as classes whose members differ person from person.

\footnotetext{
${ }^{7}$ http://matheboard.de/lexikon/Hauptseite,definition.htm

8 Tagereise: einen Tag dauernde Reise (bes. mit Pferd u. Wagen).

${ }^{9}$ Personal communication with Shou-Ren Lu.

10 The definitions of 'Chi' and 'Cun' in Shuo Wen Jie Zi (Origin of Chinese Characters), by $X U$ Shen (58-147 AD) of the Eastern Han Dynasty.

${ }^{11}$ Personal communication with Shou-Ren Lu.
} 